\title{
IFN-a2a Therapy in Two Patients with Inborn Errors of TLR3 and IRF3 Infected with SARS-CoV-2
}

\author{
Romain Lévy ${ }^{1,2,3}$ (1) P Paul Bastard ${ }^{1,2,6} \cdot$ Fanny Lanternier ${ }^{2,4}$ - Marc Lecuit ${ }^{2,4,5} \cdot$ Shen-Ying Zhang ${ }^{1,2,6}$. \\ Jean-Laurent Casanova ${ }^{1,2,6,7}$
}

Received: 4 November 2020 / Accepted: 24 November 2020 / Published online: 3 January 2021

(C) Springer Science+Business Media, LLC, part of Springer Nature 2021, corrected publication 2021

We recently reported that inborn errors of the TLR3- and IRF7-dependent production and amplification of type I interferons (IFNs) confer a predisposition to life-threatening COVID-19 pneumonia [1]. Inborn errors of eight genes were found to be causal: five from the TLR3-dependent pathway of induction (TLR3, TICAM1, UNC93B1, TBK1, IRF3), and three governing type I IFN induction and amplification (IRF7, IFNAR1, IFNAR2). These inborn errors include autosomal recessive (AR) (IRF7, IFNAR1) and autosomal dominant (AD) disorders (TLR3, TICAM1, UNC93B1, TBK1, IRF3, IRF7, IFNAR1, IFNAR2). Four of the disorders observed had not previously been described (AD UNC93B, IRF7, IFNAR1 and IFNAR2 deficiencies), whereas the other six (AR IRF7 and IFNAR1 deficiencies, and AD TLR3, TICAM1, TBK1 and IRF3 deficiencies) had previously been reported in patients with severe influenza pneumonitis, herpes simplex encephalitis (HSE), or adverse reactions to live attenuated viral vaccines [1]. These findings suggested that early type I IFN administration might be beneficial in selected

Romain Lévy

romain.levy@inserm.fr

1 Laboratory of Human Genetics of Infectious Diseases, Necker Branch, INSERM U1163, Necker Hospital for Sick Children, Paris, France

2 University of Paris, Imagine Institute, Paris, France

3 Pediatric Hematology and Immunology Unit, Necker Hospital for Sick Children, AP-HP, Paris, France

4 Necker Pasteur Center for Infectious Diseases and Tropical Medicine, Necker Hospital for Sick Children, AP-HP, Paris, France

5 Biology of Infection Unit, Institut Pasteur, Inserm U1117, Paris, France

6 St. Giles Laboratory of Human Genetics of Infectious Diseases, Rockefeller Branch, The Rockefeller University, New York, NY, USA

7 Howard Hughes Medical Institute, New York, NY 10065, USA patients known to harbor one of the inborn errors known to affect the production or amplification of type I IFN.

We recently identified two adults with a known AD IEI of type I IFN production and COVID-19. Patient 1 was a 25-yearold woman with AD TLR3 deficiency (c.1660C $>$ T; p.Pro455Ser) [2, 3]. She had two episodes of HSE as a child (at the ages of 5 and 7 years), but had since suffered no further serious viral infections [3]. Aware of her predisposition to severe COVID-19, she contacted our unit within the first 2 days of symptoms, and was admitted. She reported headaches, dyspnea, cough, fatigue, low-grade fever, and anosmia/ageusia. Neurological examination was normal. Real-time PCR (RTPCR) on a nasal swab collected on admission (day 2 of symptoms) confirmed a high viral load of SARS-Cov2 (Ct: 23). However, digital droplet PCR failed to detect the virus in the blood. Oxygen saturation was $97 \%$, and physical examination was normal, with no signs of pneumonitis. A CT scan of the lungs also revealed no signs of pneumonitis. C-reactive protein (CRP) was below the threshold of detection, and a complete blood count $(\mathrm{CBC})$ revealed mild lymphopenia $\left(0.9 \times 10^{9} / \mathrm{L}\right)$. The patient received a single subcutaneous injection of $90 \mu \mathrm{g}$ $(1.5 \mu \mathrm{g} / \mathrm{kg})$ Peg-IFN- $\alpha 2 \mathrm{a}$ (Pegasys). RT-PCR on nasal swab $36 \mathrm{~h}$ after Peg-IFN- $\alpha 2 \mathrm{a}$ administration showed that viral load had strongly decreased (Ct:35). The patient reported a partial resolution of anosmia within the next $48 \mathrm{~h}$. She was discharged on day 9 , with no symptoms other than asthenia and residual anosmia. An evaluation on day 14 was unremarkable.

Patient 2 (P2) was a 29-year-old woman with AD IRF3 deficiency (c.761-764del; p.Asp254Glyfs*3) who had suffered recurrent mucocutaneous HSV-1 infections, at intervals of about 1 month, since the age of 23 years. She was admitted 7 days after the onset of symptoms, including fever, fatigue, cough, odynophagia, headaches, diarrhea, and anosmia/ ageusia beginning $24 \mathrm{~h}$ before admission. Physical examination was normal and oxygen saturation was 99\%. RT-PCR on a nasal swab collected on admission confirmed the presence of a high viral load of SARS-Cov2 (Ct: 21). CBC was normal, 
and CRP was undetectable. A CT scan showed ground-glass opacities in her left lower lobe with posterior predilection affecting about $10 \%$ of the lungs compatible with COVID19. The patient received $80 \mu \mathrm{g}(1.5 \mu \mathrm{g} / \mathrm{kg})$ Peg-IFN- $\alpha 2 \mathrm{a}$ administered subcutaneously. P2 reported a resolution of symptoms, including anosmia/ageusia, within $48 \mathrm{~h}$ of IFN administration. She was discharged on day 12 .

We report here the safety of a single subcutaneous injection of Peg-IFN- $\alpha 2 \mathrm{a}$ in two patients with IEIs affecting the production of type I IFNs associated with a predisposition to severe COVID-19 [1]. The rationale for proposing IFN- $\alpha 2 a$ treatment to these two patients was based on (i) the impairment of type I IFN production by both AD TLR3 and IRF3 deficiencies [2-5]; (ii) the development of severe COVID-19, sometimes with a fatal outcome, in some patients with IEIs affecting type I IFN production [1]; (iii) the anosmia/ageusia in both patients, whose IEI predispose to HSE, and the tropism of SARS-Cov2 for the central nervous system, which together stressed a risk of neurological manifestations of COVID-19; (iv) the high susceptibility of TLR3-deficient fibroblasts to SARS-Cov2 in vitro, a phenotype rescued by exogenous type I IFN; (v) the diagnosis of these two patients with COVID-19 at a very early stage of clinical manifestations, placing both patients in the so-called first phase of COVID-19; and (vi) the known safety profile of a single dose of Peg-IFN- $\alpha 2 a$. Our findings cannot be considered a formal demonstration of the efficacy of IFN- $\alpha 2$ a therapy for COVID-19, but both patients reported a rapid resolution of the symptoms and signs present at admission following the administration of Peg-IFN- $\alpha 2$ a. This observation suggests that patients with known IEIs affecting the TLR3- and IRF7-dependent production and amplification of type I IFN signaling may benefit from the very early administration of type I IFN. Furthermore, this observation suggests that, in trials of type I IFN-based regimens for treating SARS-CoV-2-infected patients at risk of developing severe disease, the earliest possible administration should be considered.

Acknowledgments We thank the members of the Laboratory of Human Genetics of Infectious Diseases: Laurent Abel, Aurélie Cobat, Qian Zhang, Emmanuelle Jouanguy, Lazaro Lorenzo-Diaz and Yelena Nemirovskaya for their expertise; the members of the Necker Pasteur
Center for Infectious Diseases and Tropical Medicine, and the Adult hematology unit in Necker hospital for Sick Children in Paris, for their medical expertise and assistance: Olivier Lortholary, Olivier Hermine, Claire Aguilar, Emmanuel Lafont, Fanny Alby-Laurent; the members of the Center for the Study of Primary Immunodeficiencies in Necker hospital for Sick Children in Paris: Jacinta Bustamante, Mathieu Fusaro for their assistance. The Laboratory of Human Genetics of Infectious Diseases is supported by the Howard Hughes Medical Institute, the Rockefeller University, the St. Giles Foundation, the National Institutes of Health (NIH) (R01AI088364), the National Center for Advancing Translational Sciences (NCATS), NIH Clinical and Translational Science Award (CTSA) program (UL1 TR001866), a Fast Grant from Emergent Ventures, Mercatus Center at George Mason University, the Yale Center for Mendelian Genomics and the GSP Coordinating Center funded by the National Human Genome Research Institute (NHGRI) (UM1HG006504 and U24HG008956), the Fisher Center for Alzheimer's Research Foundation, the Meyer Foundation, the Square Foundation, the French National Research Agency (ANR) under the "Investments for the Future" program (ANR-10-IAHU-01), the Integrative Biology of Emerging Infectious Diseases Laboratory of Excellence (ANR-10-LABX-62-IBEID), the French Foundation for Medical Research (FRM) (EQU201903007798), the FRM and ANR GENCOVID project, ANRS-COV05, Grandir - Fonds de solidarité pour l'enfance, the SCOR Corporate Foundation for Science, Institut National de la Santé et de la Recherche Médicale (INSERM) and the University of Paris.

\section{References}

1. Zhang Q, Bastard P, Liu Z, Le Pen J, Moncada-Velez M, Chen J, et al. Inborn errors of type I IFN immunity in patients with lifethreatening COVID-19. Science. 2020;370:eabd4570.

2. Lim HK, Huang SXL, Chen J, Kerner G, Gilliaux O, Bastard P, et al. Severe influenza pneumonitis in children with inherited TLR3 deficiency. J Exp Med. 2019;216:2038-56.

3. Zhang S-Y, Jouanguy E, Ugolini S, Smahi A, Elain G, Romero P, et al. TLR3 deficiency in patients with herpes simplex encephalitis. Science. 2007;317:1522-7.

4. Andersen LL, Mørk N, Reinert LS, Kofod-Olsen E, Narita R, Jørgensen SE, et al. Functional IRF3 deficiency in a patient with herpes simplex encephalitis. J Exp Med. 2015;212:1371-9.

5. Thomsen MM, Jørgensen SE, Storgaard M, Kristensen LS, Gjedsted $\mathrm{J}$, Christiansen $\mathrm{M}$, et al. Identification of an IRF3 variant and defective antiviral interferon responses in a patient with severe influenza. Eur J Immunol. 2019;49:2111-4.

Publisher's Note Springer Nature remains neutral with regard to jurisdictional claims in published maps and institutional affiliations. 